\title{
Microwave-assisted generation of carbazolyl nitrenium cation
}

\author{
Dariusz Bogdal \\ Department of Chemistry, Politechnika Krakowska ul. Warszawska 24, 31-155 Krakow, Poland \\ E-mail:pcbogdal@cyf-kr.edu.pl
}

\section{Dedicated to Professor Rudolph A. Abramovitch on the occasion of his $70^{\text {th }}$ birthday (received 02 May 01; accepted 15 Oct 01; published on the web 23 Oct 01)}

\begin{abstract}
Carbazolyl nitrenium cations were generated during microwave irradiation of 1-carbazol-9-yl2,4,6-triphenylpyridinium tetrafluoroborate in trifluoroethanol- and TFA - arenes mixtures to give desired electrophilic substitution reaction products. Unlike under conventional conditions (thermolysis and photolysis) the parent amine, carbazole, and other side product were detected only in trace amounts.
\end{abstract}

Keywords: Carbazole, arene, microwave, nitrenium, pyridinium

\section{Introduction}

The chemistry of nitrenium ions has been broadly reported since the investigation of Abramovitch and his group., ${ }^{1,2}$ These species that contain positively charged nitrogen atom and can possess either the singlet and triplet ground states receive special attention because they are potential intermediates in carcinogenic process. ${ }^{3}$ On the other hand, nitrenium ions have potential synthetic applications owing to direct amination (replacement of $\mathrm{H}$ by $\mathrm{NR}_{2}$ ) of aromatic compounds. ${ }^{4,5}$

Recently, we have reported that a carbazolyl nitrenium ion could be successfully generated during the thermolysis and/or photolysis of 1-carbazol-9-yl-2,4,6-triphenylpyridinium Py+-Cz tetrafluoroborate in trifluoroethanol- and/or TFA - mesitylene mixtures. ${ }^{6}$ The investigations showed that carbazolyl nitrenium ion exhibits singlet state in the ground state so in order to react it with aromatic compounds, like mesitylene, high temperature procedures have to be employed. The disadvantages of the thermolysis reaction of pyridinium salts are a long reaction time, application of inert atmosphere, and tedious preparation of reaction mixtures (i.e., vacuumed sealed vessels).

In the last few years there has been a growing interest in the use of microwave irradiation to organic synthesis. ${ }^{7}$ The use of such reaction conditions reveals several features like: a short 
reaction time compared to conventional heating, ease of work-up after a reaction, and reduction in the usual thermal degradation and better selectivity. Since we have shown that many different reactions could be achieved under microwave conditions, ${ }^{8}$ we decided to explore the use of microwaves to the generation of a carbazolyl nitrenium ion from 1-carbazol-9-yl-2,4,6triphenylpyridinium $\mathbf{P y}+\mathbf{- C z}$ tetrafluoroborate.

\section{Results and Discussion}

The starting pyridium salt $\mathbf{P y +} \mathbf{- C z}$ was readily prepared in the reaction of 9-aminocarbazole9 and 2,4,6-triphenylpyrylium tetrafluoroborate in a dry ethanol solution (Scheme 1). ${ }^{6}$

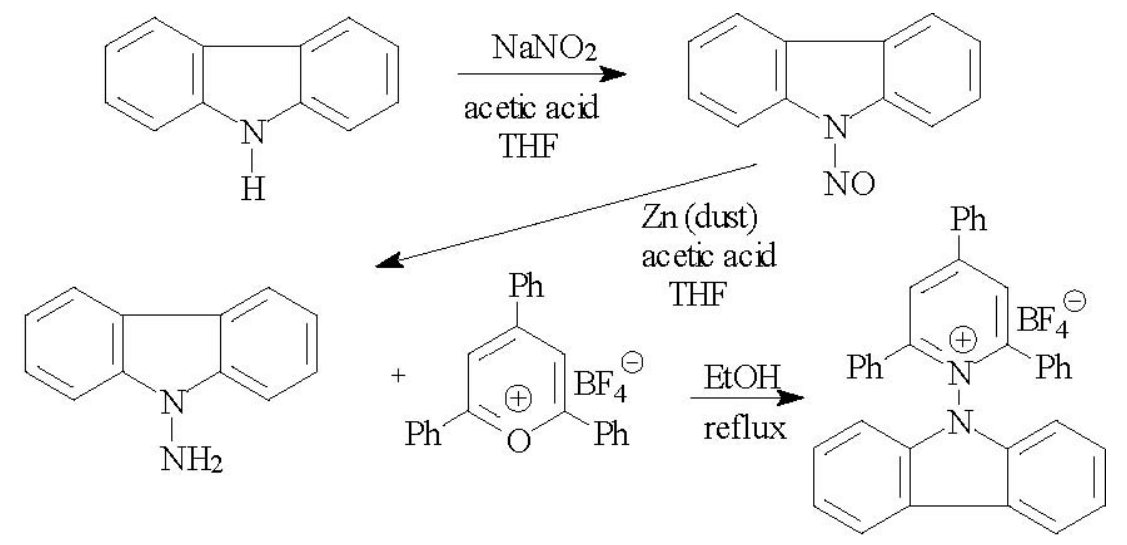

\section{Scheme 1}

In a typical experiment, the pyridinium salt $\mathrm{Py}+-\mathrm{Cz}$ was added to an aromatic compound together with trifluoroethanol (TFE) or TFA. The mixture was then irradiated in an open vessel in Prolabo Sythwave 402 microwave reactor for 15 to 20 min while temperature was monitored with Nortech ReFlex fiber-optic system. Upon completion of the reaction, aqueous potassium carbonate was added to the mixture till the $\mathrm{pH}$ of the solution reached ${ }^{7}$. The organic layer was extracted with ethyl acetate, and the yield was determined by GC/MS analysis. The products were identified by comparison with original samples that had been obtained in our previous study on the generation of carbazolyl cation under conventional conditions. ${ }^{6}$ The conditions and results of the reactions under microwave irradiation are summarized in Table 1. 
Table 1. The GC/MS analysis after microwave irradiation of pyridinium salt in TFA- and TFEaromatic compounds mixtures ${ }^{\text {a) }}$

\begin{tabular}{|c|c|c|c|c|c|c|}
\hline Substrate & $\begin{array}{c}\text { Amount } \\
\text { [ml] }\end{array}$ & $\begin{array}{l}\text { TFA } \\
{[\mathrm{ml}]}\end{array}$ & $\begin{array}{l}\text { TFE } \\
{[\mathrm{ml}]}\end{array}$ & $\begin{array}{l}\text { Time } \\
{[\mathrm{min}]}\end{array}$ & $\begin{array}{c}\text { Products } \\
\text { (yield [\%]) }^{\text {b) }}\end{array}$ & $\begin{array}{c}\text { Final temp. } \\
{\left[{ }^{\circ} \mathrm{C}\right]}\end{array}$ \\
\hline Mesitylene & 7.0 & 1.5 & & 16 & 1 (70), 2 (30) & 171 \\
\hline Mesitylene & 7.0 & & 2.0 & 16 & 1 (55), 2 (45) & 151 \\
\hline Mesitylene & 8.0 & 10 drops & & 20 & 1 (64), 2 (36) & 166 \\
\hline p-Xylene & 6.0 & 1.5 & & 20 & 7 (20), 2 (80) & 142 \\
\hline p-Xylene & 6.0 & & 2.0 & 20 & 7 (5), 2 (95) & 136 \\
\hline Toluene & 7.0 & 1.5 & & 20 & 8 (1), 2 (99) & 115 \\
\hline Anisole & 6.0 & 1.5 & & 16 & 4 (36), 3 (20), 2 (42) & 152 \\
\hline Anisole & 6.0 & & 1.0 & 16 & 4 (29), 3 (13), 2 (56) & 158 \\
\hline Anisole & 7.0 & & & 20 & 4 (30), 3 (17), 2 (52) & 166 \\
\hline
\end{tabular}

${ }^{a}$ always $100 \mathrm{mg}$ of the pyridinium salt $\mathbf{P y}+\mathbf{- C z}$ was taken to the reaction; ${ }^{\mathrm{b}}$ all the yields are given after GC/MS analysis

The microwave irradiation of the pyridinium salt $\mathbf{P y}+\mathbf{C z}$ in a mesitylene solution in the presence of TFA as well as TFE resulted in the synthesis of 9-mesitylcarbazole $\mathbf{1}$ together with 2,4,6-triphenylpyridine 2, which is the expected by-product of the decomposition of the pyridinium salt (Scheme 2).
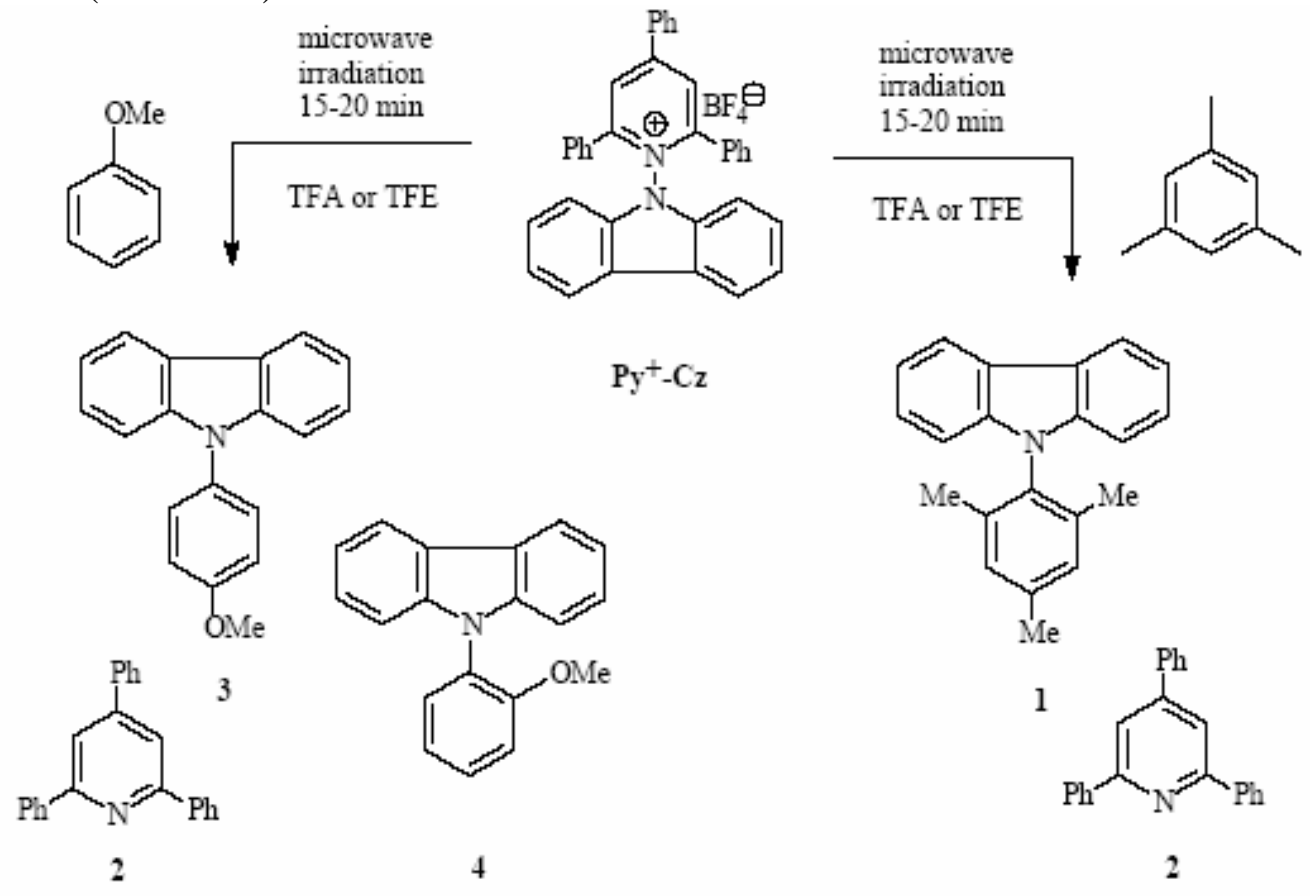

\section{Scheme 2}


9-Mesitylcarbazole 1 was produced by electrophilic substitution reaction on the mesitylene aromatic ring. Unlike under conventional conditions for thermolysis and photolysis reactions, other compounds like the non-symmetrical mesitylene dimer 5 (Fig. 2) and parent amine, carbazole 6, were detected only in trace amounts. A typical chromatogram run after the reaction is shown on Figure 1. The retention times of 9-mesitylcarbazole 1 and 2,4,6-triphenylpyridine 2 are ca. 17 and 31 min., respectively.

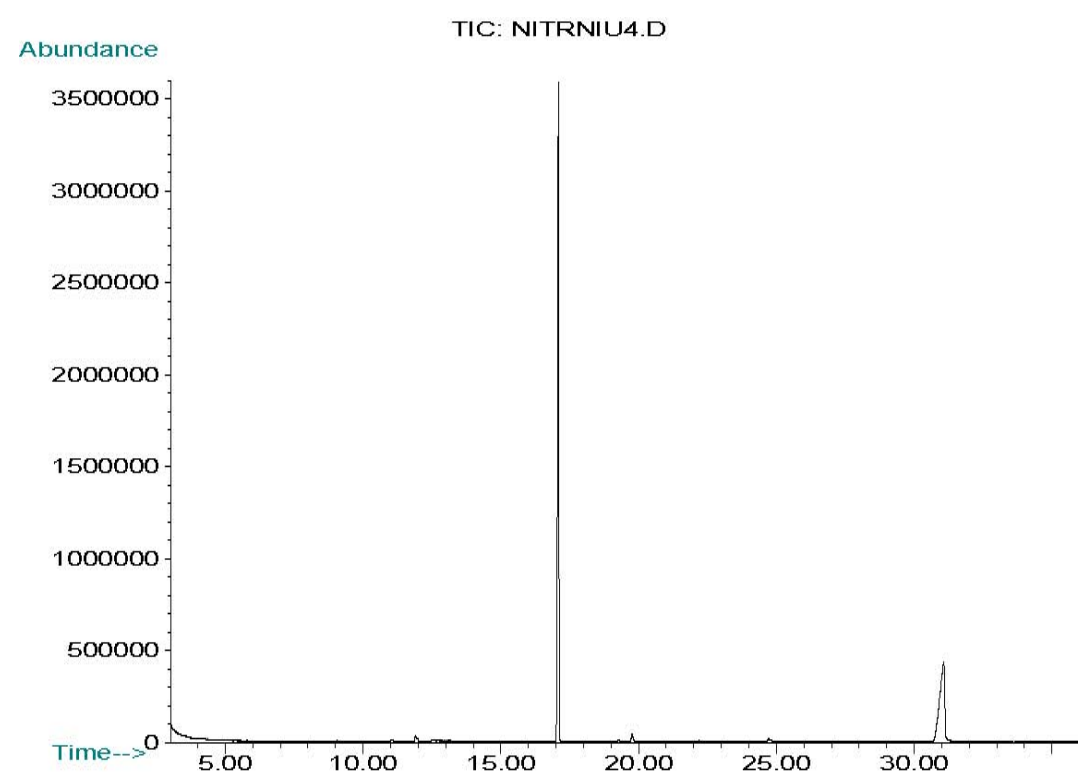

Figure 1. The GC/MS chromatogram of the reaction mixture (mesitylene and $\mathbf{P y +} \mathbf{y} \mathbf{z}$ )

The reaction occurred with better yield in much shorter time (i.e., $16 \mathrm{~min}$ ) in comparison with the thermolysis reactions $(8 \mathrm{~h}){ }^{6}{ }^{6}$ Moreover, the reaction conditions were simplified, and there was no need for the use of inert atmosphere during the synthesis. The only preparation work prior to the synthesis was the distillation of mesitylene, which was in turn kept above molecular sieves. It was found that in order to achieve full decomposition of the pyridinium salt, the final reaction temperature has to exceed $150^{\circ} \mathrm{C}$, otherwise 9-mesitylenecarbazole 1 was obtained with substantially smaller yields. For the same reasons, in case of p-xylene and toluene the expected products $\mathbf{7}$ and $\mathbf{8}$ were obtained in small and trace amounts, respectively.

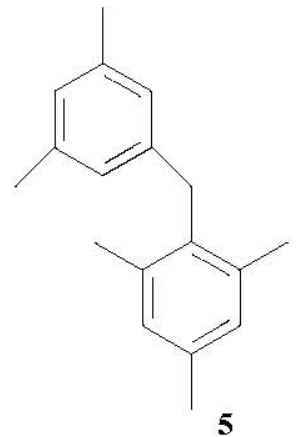

5

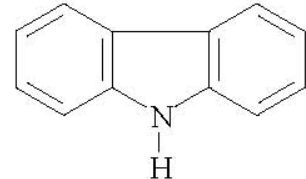

6

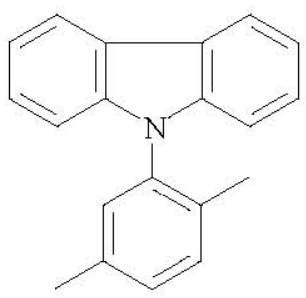

7

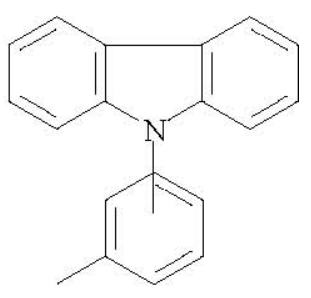

8 
Like in the case of mesitylene, the reaction of the pyridinium salt Py+-Cz with anisole under microwave irradiation afforded in 9:5 ratio two expected compounds i.e., 9-(omethoxyphenyl)carbazole 4 and 9-(p-methoxyphenyl)carbazole 3, which are the products of electrophilic substitution reaction in the anisole aromatic ring (Scheme 2). Furthermore, carbazole 6 (i.e., the parent amine of the generated nitrenium cation), and mesitylene dimer 5 were again detected in trace amounts. The retention times of 9-( $p$-methoxyphenyl)carbazole 3, 9(o-methoxyphenyl)carbazole 4, and 2,4,6-triphenyl-pyridine 2 are ca. 18, 21 and 31 min., respectively (Figure 2).

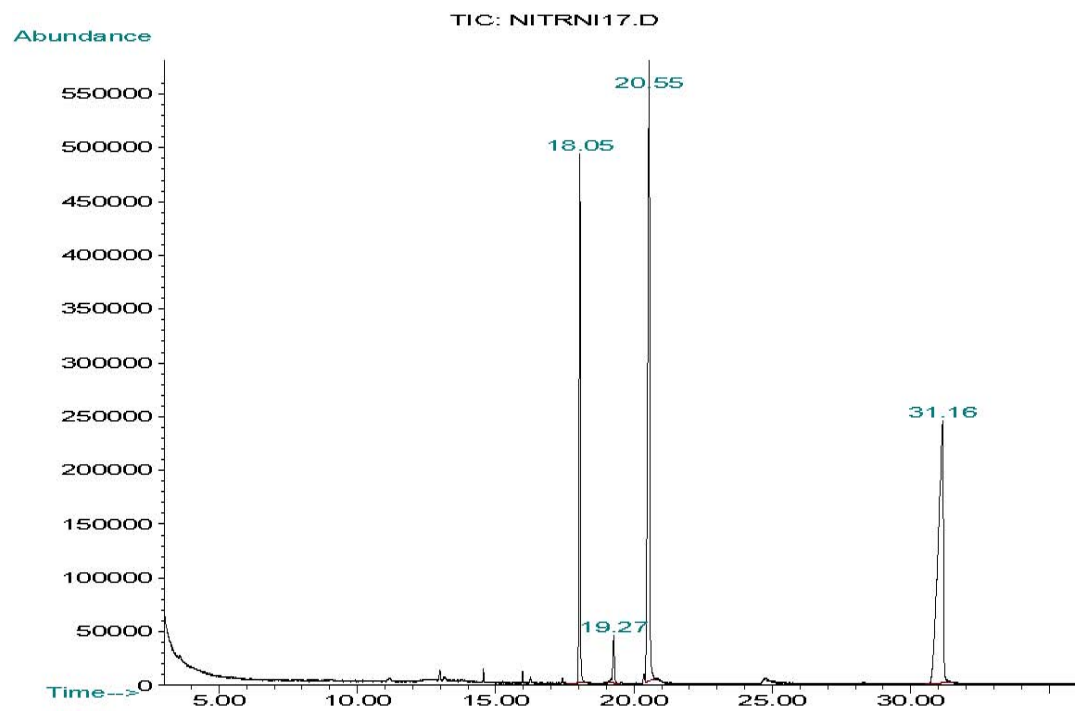

Figure 2. The CG/MS chromatogram of the final reaction mixture (anisole and Py+-Cz) after microwave irradiation

In the next stage of investigation, strongly acidic conditions were changed to neutral conditions, and trifluoroethanol (TFE) was added to the reaction mixtures instead of TFA. The yields of 1, 3, and $\mathbf{4}$ were slightly reduced, while carbazole $\mathbf{6}$ and mesitylene dimer $\mathbf{5}$ were still recorded in trace amounts. More interesting was the reaction in the absence of TFA or trifluoroethanol. In the case of mesitylene, the reaction mixture did not absorb microwaves, reaching $65^{\circ} \mathrm{C}$ after $10 \mathrm{~min}$ of irradiation with $30 \%$ of the reactor power. After the addition of 10 drops of TFA, temperature rose to $150^{\circ} \mathrm{C}$ and $165^{\circ} \mathrm{C}$ in 10 and $15 \mathrm{~min}$, respectively. Eventually, 9-mesitylcarbazole 1 was isolated with 64\% yield. In opposite to mesitylene, anisole absorbs microwaves, and it was possible to carry out the reaction without addition on any polar substance that couples strongly with microwaves. The temperature increase was fast to reach $160^{\circ} \mathrm{C}$ in 6 min, and the reaction was achieved in 20 min under microwave irradiation.

In conclusion, the carbazolyl nitrenium cation possesses singlet state in the ground state, ${ }^{6}$ and, therefore, it can be successfully generated under microwave irradiation. The main advantages of the microwave procedure are a shorter reaction time reduced from hours to minutes, and much more favourable distribution of the reaction products. Unlike the reaction 
under conventional conditions, this synthesis gives only one by-product, triphenylpyridine, which makes the work-up procedure less tedious. The parent amine of the nitrenium cation, carbazole, was always detected in trace amounts.

\section{Experimental Section}

General Procedures. IR spectra were recorded using a Bio-Rad FTS 165 spectrophotometer. ${ }^{1} \mathrm{H}-\mathrm{NMR}$ spectra were taken with a Tesla $487 \mathrm{C}$ instrument, TMS being used as a internal standard; the chemical shifts are expressed in d-values downfield from TMS. MS spectra were recorded with a Hewlett-Packard GC/MS 5985 spectrometer. Elemental analyses were performed on a Perkin-Elmer 2400 microanalyser. Melting points measured on a Boetius-PHMK 05 microscope plates are uncorrected. TLC was performed with Aluminum oxide 60F 254 Type E (5713) plates (Merck). Ethyl acetate, toluene, and p-xylene were purified by standard methods before use. Mesitylene, trifluoroacetic acid and trifluoroethanol were purified by distillation. The following compounds were reagent grade and used without further purification: carbazole (Fluka), 2,2,2-trifluoroethanol (Fluka), 2,4,6-triphenylpyrylium tetrafluoroborate (Aldrich). 1Carbazol-9-yl-2,4,6-triphenylpyridinium tetrafluoroborate $(\mathrm{Py}+\mathrm{-Cz})$ was prepared according to the procedure given in the previous paper,6 and all the compounds were characterized by the comparison with the sample prepared under conventional procedure given the same paper.

Microwave irradiation of 1-carbazol-9-yl-2,4,6-triphenylpyridinium tetrafluoroborate Py+Cz: Microwave irradiation of 1-(carbazol-9-yl)-2,4,6-triphenylpyridinium tetrafluoroborate $\mathbf{P y + -}$ Cz was carried out in a open vessel in Prolabo Synthwave 402 microwave reactor. The vessel was placed inside the reactor and rotated in order to ensure the homogeneity of microwave field. The temperature was detected with Nortech ReFlex fibber-optic thermometer. The detail conditions of all the reaction are given in Table 1 . After the reaction, aqueous potassium carbonate was added to the mixture till the $\mathrm{pH}$ of the solution exceeded 7. The organic layer was extracted with ethyl acetate. The progress of the reactions was monitored by TLC while the yields were determined by GC/MS analysis.

\section{Acknowledgements}

This work was undertaken as part of the EU sponsored D10 COST Program (Innovative Methods and Techniques for Chemical Transformations). 


\section{References and Notes}

1. Abramovitch R. A.; Jeyaraman, R. In Azides and Nitrenes: Reactivity and Utility. Scriven, E. F. V. Ed. Academic Press: Orlando, FL, 1984.

2. (a) Abramovitch, R. A.; Bartnik, R.; Cooper, M.; Dassanayake, N. L.; Hwang, H. Y.; Inbasekaran M. N.; Rusek, G. J. Org. Chem. 1982, 47, 4817. (b) Abramovitch, R. A.; Beckert, J. M.; Pennington, W. T. J. Chem. Soc., Perkin Trans 1 1991, 1761. (c) Abramovitch, R. A.; Gibson, Jr., H. H.; Nguyen, N. T.; Olivella, S.; Solé, A. Tetrahedron Lett. 1994, 35, 2321. (d) Abramovitch, R. A.; Evertz, K.; Huttner, G.; Gibson, Jr. H. H.; Weems, H. G. J. Chem. Soc., Chem. Commun 1988, 325.

3. Novak, M.; Kennedy, S. A. J. Am. Chem. Soc. 1995, 117, 574.

4. (a) Takeuchi, H.; Koyama, K. J. Chem. Soc., Chem. Commun. 1981, 203. (b) Takeuchi, H.; Takano, K.; Koyama, K. J. Chem. Soc., Chem. Commun. 1982, 1254.

5. (a) Moran, R. J.; Cramer, C.; Falvey, D. E. J. Org. Chem. 1997, 62, 2742. (b) Moran, R. J.; Falvey, D. E. J. Am. Chem. Soc. 1996, 118, 8965.

6. Bogdal, D. Heterocycles 2000, 53, 2679.

7. For relevant papers and reviews on microwave assisted chemical reactions see: (a) Abramovitch, R. A. Org. Prep. Proc. Int. 1991, 23, 683. (b) Majetich, G.; Hicks, R. J. Radiat. Phys. Chem. 1995, 45, 567. (c) Caddick, S. Tetrahedron 1995, 51, 10403. (d) Strauss, C. R.; Trainor, R.W. Aust. J. Chem. 1995, 48, 1665. (e) Varma, R. S. Green Chemistry 1999, 1, 43. (f) Loupy, A.; Petit, A.; Hamelin, J.; Texier-Boullet, F.; Jacquault, P.; Mathe, D. Synthesis 1998, 1213. (g) Bogdal, D. Wiad. Chem. 1999, 53, 66.

8. (a) Abramovitch, R. A.; Shi, Q.; Bogdal, D. Synth. Commun. 1995, 25, 1. (b) Bogdal, D.; Pielichowski, J.; Boron, A. Synlett 1996, 873. (c) Bogdal, D.; Pielichowski, J.; Jaskot. K. Heterocycles 1997, 45, 715. (d) Bogdal, D.; Pielichowski, J.; Jaskot, K. Org. Prep. Proc. Int. 1998, 30, 427. (e) Bogdal D., J. Chem. Res. (S) 1998, 468. (f) Bogdal, D.; Lukasiewicz, M. Synlett 2000, 143. (g) Bogdal, D.; Warzala, M. Tetrahedron 2000, 56, 8769.

9. Kyziol, J.; Tarnawski, J. Rev. Roum. Chim. 1980, 25, 721. 